\title{
C-spaces, generalized geometry and double field theory
}

\section{G. Papadopoulos}

Department of Mathematics, King's College London, Strand, London WC2R 2LS, U.K.

E-mail: george.papadopoulos@kcl.ac.uk

ABStRaCT: We construct a C-space associated with every closed 3-form on a spacetime $M$ and show that it depends on the class of the form in $H^{3}(M, \mathbb{Z})$. We also demonstrate that $\mathrm{C}$-spaces have a relation to generalized geometry and to gerbes. C-spaces are constructed after introducing additional coordinates at the open sets and at their double overlaps of a spacetime generalizing the standard construction of Kaluza-Klein spaces for 2-forms. Cspaces may not be manifolds and satisfy the topological geometrization condition. Double spaces arise as local subspaces of C-spaces that cannot be globally extended. This indicates that for the global definition of double field theories additional coordinates are needed. We explore several other aspect of C-spaces like their topology and relation to Whitehead towers, and also describe the construction of C-spaces for closed k-forms.

KEYwords: String Duality, Bosonic Strings, Superstring Vacua

ARXIV EPRINT: 1412.1146 


\section{Contents}

1 Introduction 1

2 C-spaces for closed 3-forms $\quad 4$

2.1 C-spaces for closed 2-forms 4

2.2 Patching C-spaces for closed 3-forms 5

2.3 The $y^{1}$ coordinates 6

2.4 Dependence on $\omega^{3} \quad 6$

$\begin{array}{lll}2.5 & \text { Dependence on the cover } & 7\end{array}$

$\begin{array}{ll}2.6 & \text { Topological geometrization condition } \\ \end{array}$

3 Relation to gerbes and generalized geometry $\quad 8$

3.1 Gerbes 8

$\begin{array}{lll}3.2 & \text { Generalized geometry } & 9\end{array}$

3.2.1 Closed 2-forms 9

3.2.2 Closed 3-forms 9

4 Some topological aspects of $C_{M}^{\left[\omega^{3}\right]}$ and an example $\quad 10$

$\begin{array}{lll}4.1 \text { Topological aspects } & 10\end{array}$

$\begin{array}{ll}\text { 4.2 The C-space of 3-torus with a 3-form flux } & 11\end{array}$

$5 \quad$ DFT on double manifolds $\quad 12$

$\begin{array}{lll}5.1 & \text { Revisiting the patching of double manifolds } & 12\end{array}$

$\begin{array}{lll}5.2 & \text { Relation of double spaces to C-spaces } & 14\end{array}$

6 C-spaces for closed k-forms $\quad 14$

6.1 The construction of $C_{M}^{\left[\omega^{k}\right]} \quad 14$

$\begin{array}{lll}6.2 \text { Applications } & 15\end{array}$

$\begin{array}{llr}7 & \text { Whitehead towers and } C_{M}^{\left[\omega^{k}\right]} & 16\end{array}$

$\begin{array}{lll}8 & \text { Concluding remarks } & 17\end{array}$

\section{Introduction}

Double field theory (DFT) has been introduced to provide a geometric interpretation of the T-duality symmetries and to describe string theory in a T-duality covariant way, see [1-4] for early works and [5]-[14] for more recent developments. More general proposals ${ }^{1}$ include

\footnotetext{
${ }^{1}$ See $[15]$ for an early work on the geometrization of dualities.
} 
$E_{11}[16,17]$ and exceptional field theories, see eg [18]-[30], and reviews [31-33] and references with. For the construction of DFT, the spacetime $M$ is enhanced with additional coordinates, leading to a double space $D_{M}$ which has dimension twice that of spacetime. So far the construction of local actions relies on two ingredients. First, the use of infinitesimal transformations to prove invariance, and second the application of the strong section condition. These infinitesimal transformations combine the spacetime diffeomorphisms and the gauge transformations of the $B$-field that act on a generalized metric. This generalized metric is constructed from both the spacetime metric and the $B$-field. This is interpreted as a geometrization of $B$-field. The strong section condition in effect restricts the fields and their infinitesimal transformations to dependent on either the spacetime or dual coordinates. More recently several suggestions have been made to integrate the infinitesimal transformations of DFT leading to the construction of finite transformations for the double spaces and for the associated fields [33-36]. Another suggestion is to employ a non-trivial split metric on the extended spaces [37]. Similar results also hold for the exceptional field theories, however see also [38, 39].

The global definition of DFTs remains an open problem. Using the solution of the strong section condition for the spacetime presented in [33, 34], it has been shown in [40] that the patching of double spaces ${ }^{2}$ constructed is consistent if and only if the 3-form field strength is exact. In section 5 , we shall strengthen this statement. The C-spaces that we propose below resolve this global patching problem.

To identify the spaces which can implement the geometrization of the $B$-field in the context of DFT, it has also been argued in [40] that one necessary ingredient is the topological geometrization condition. This can be stated as follows: given a manifold $M$, eg spacetime, and a closed k-form $\omega^{k}$, a space ${ }^{3} C_{M}$ satisfies the topological geometrization condition, if and only if there is a projection $\pi: C_{M} \rightarrow M$ such that $\pi^{*} \omega^{k}$ represents the trivial class in $H^{k}\left(C_{M}\right)$.

Given $M$ and $\omega^{k}$, this definition does not uniquely specify $C_{M}$. There are several constructions of $\mathrm{C}$-spaces via K-theory and homotopy theory. The latter applies for any manifold and for any form of any degree. The standard examples of C-spaces are circle bundles over $M$ which satisfy the topological geometrization property for closed 2-forms, and implement the geometrization of the Maxwell fields in the context of Kaluza-Klein theory.

In this paper, a construction of C-spaces, $C_{M}^{\left[\omega^{3}\right]}$, is proposed for every closed 3-form, $\omega^{3}$, on a manifold $M$ provided that $\left[\omega^{3}\right] \in H^{3}(M, \mathbb{Z})$ which is suitable for applications in DFT. The construction involves the introduction of new coordinates associated with the gauge transformations of the transition functions of $\omega^{3}$ at double overlaps with respect to both the Čech and de Rham differentials. This leads to an additional

- (local) 1-form coordinate $y^{1}$ for every open set of spacetime, as for double spaces, ${ }^{4}$

- and a new angular coordinate $\theta$ at every double intersection of two open sets.

\footnotetext{
${ }^{2}$ Examples of double spaces have been investigated in [40] from the patching point of view and they have been found to depend on the choice of atlas. Therefore they are not general covariant.

${ }^{3}$ The spaces that satisfy the topological geometrization property have been called C-spaces because the topological charge carried by $\omega^{k}$ is stored in the transition functions of $C$.

${ }^{4}$ However, we shall demonstrate that $y^{1}$ and the corresponding coordinates for double spaces transform differently.
} 
Exploring the consistency of the patching conditions given in (2.6) at triple and 4-fold overlaps, it leads to the requirement that $\omega^{3}$ must represent a class in $H^{3}(M, \mathbb{Z})$ as expected from the Dirac quantization condition. In addition, it is demonstrated that $C_{M}^{\left[\omega^{3}\right]}$ depends on $\left[\omega^{3}\right]$, ie it is independent from the choice of a representative of the cohomology class $\left[\omega^{3}\right]$. From construction is apparent that $C_{M}^{\left[\omega^{3}\right]}$ are not manifolds, in particular they may not have a well-defined dimension. Nevertheless they can be described in some detail using the transition functions and the additional coordinates. Furthermore, one can show that $C_{M}^{\left[\omega^{3}\right]}$ satisfies the topological geometrization condition.

This construction of C-spaces for closed 3-forms is related to gerbes. In particular, we explain how from $C_{M}^{\left[\omega^{3}\right]}$ one can construct the gerbe transition functions that arise in the approach of [41]. However the construction of $C_{M}^{\left[\omega^{3}\right]}$ involves the open sets and their double overlaps, as well as the triple and 4-fold overlaps, in an essential way, and the emphasis is on the object itself rather than its transition functions on $M$. This is more close in spirit to the definition of gerbes in terms of sheafs [42] but without the complications of category theory. Furthermore, the construction of $C_{M}^{\left[\omega^{3}\right]}$ gives a geometric interpretation into generalized geometry on $M$ as described by Hitchin and Gualtieri [43-45]. In particular we shall show that the twisted Courant bracket on the spacetime can be derived from a Courant bracket on $C_{M}^{\left[\omega^{3}\right]}$. As result one can define a generalized metric and carry out generalized differential geometry calculus on $M$.

To get some insight into the topological structure of $C_{M}^{\left[\omega^{3}\right]}$, we consider the nerve of the good cover of $M$ which provides a chain complex description of $M$. We find that every 2-simplex in the nerve of $M$ together with the new angular coordinates give rise to a $\mathbb{C} P^{2}$ in $C_{M}^{\left[\omega^{3}\right]}$. We use this to raise the question whether this construction of $C_{M}^{\left[\omega^{3}\right]}$ is related to Whitehead towers. Furthermore, we construct, $C_{T^{3}}^{\left[\omega^{3}\right]}$, which is the C-space of 3-torus with a 3 -form flux. We demonstrate that $C_{T^{3}}^{\left[\omega^{3}\right]}$ resolves the patching problems of the double space construction of [33] for this model.

To elucidate the relation between C-spaces and double spaces, we revisit the global properties of the double spaces. We show that the mere use of the strong section condition, ie without invoking any information about the transformation of the generalized fields, together with the requirement of the general covariance of the spacetime imply that the double space must be diffeomorphic to $T^{*} M$. Such a space cannot satisfy the topological geometrization property and also is in conflict with established examples of T-dual pairs. Moreover if the transition functions of the B-field are related in a linear way to those of the dual coordinates, then the 3 -form flux is exact.

Furthermore, we demonstrate that the C-spaces $C_{M}^{\left[\omega^{3}\right]}$ locally include the double spaces. In particular, the double spaces arise as subspaces of $C_{M}^{\left[\omega^{3}\right]}$ after taking the new angular coordinates $\theta$ to vanish. This can be consistently done only at appropriate open sets and not globally over the whole spacetime $M$. Therefore double spaces can only provide a local description DFTs, ie on a patch of $M$. For the global definition of DFTs over $M$ additional coordinates are required.

The construction of C-spaces, $C_{M}^{\left[\omega^{k}\right]}$, can be generalized to every k-form, $\omega^{k}$, which represents a class in $H^{k}(M, \mathbb{Z})$. This proceeds in a similar way to that of $C_{M}^{\left[\omega^{3}\right]}$. However, the 
construction of $C_{M}^{\left[\omega^{k}\right]}$ requires the presence of additional coordinates which are introduced at the multiple intersections of open sets of $M$. The properties of $C_{M}^{\left[\omega^{k}\right]}$ are also similar, ie $C_{M}^{\left[\omega^{k}\right]}$ satisfy the topological geometrization condition and depend on the class of $\omega^{k}$ in $H^{k}(M, \mathbb{Z})$. The extended space associated with a $\mathrm{k}$-form, which is the generalization of a double space for $k>3$, can be seen as a local subspace of $C_{M}^{\left[\omega^{k}\right]}$. This again indicates that more coordinates are need for the global description of exceptional field theories.

There is a construction of C-spaces in the context of homotopy theory using Whitehead towers. Here we revisit the theory and point out that the Whitehead towers construction for 2 -forms coincides, up to homotopy, with the standard circle bundle construction of KaluzaKlein spaces. Then we review some of the properties of Whitehead towers construction for closed 3-forms and ask the question how these are related to $C_{M}^{\left[\omega^{3}\right]}$ spaces. We also argue that the total space $X_{3}$ of the Whitehead fibration $\mathbb{C} P^{\infty} \rightarrow X_{3} \rightarrow S^{3}$ provides a homotopy description of the gerbe associated with the generator of $H^{3}\left(S^{3}, \mathbb{Z}\right)$.

This paper has been organized as follows. In section 2 , we describe the construction of $C_{M}^{\left[\omega^{3}\right]}$. In section 3, we explain the relation of $C_{M}^{\left[\omega^{3}\right]}$ to generalized geometry and gerbes. In section 4 , we investigate some of the topological properties of $C_{M}^{\left[\omega^{3}\right]}$ and present the 3-torus with 3-form flux C-space. In section 5, we explore the applications of C-spaces to DFT. In section 6 , we construct $\mathrm{C}$-spaces for closed $\mathrm{k}$-forms. In section 7 , we explore the relation between C-spaces and Whitehead towers, and in section 8, we give our conclusions.

\section{C-spaces for closed 3-forms}

\section{$2.1 \quad$ C-spaces for closed 2-forms}

Before, we proceed to give the patching conditions of C-spaces associated with closed 3forms, let us briefly review the standard Kaluza-Klein space, $C_{M}^{\left[\omega^{2}\right]}$, for 2-forms. Let $M$ be a manifold and $\left\{U_{\alpha}\right\}_{\alpha \in I}$ be a good $\operatorname{cover}^{5}$ of $M$, for the precise definition see eg [46] page 42. Moreover suppose that $\omega^{2}$ represents a class in $H^{2}(M, \mathbb{R})$. Then within the Cech-de Rham theory applying the Poincaré lemma on the open sets $U_{\alpha}$ as well as their $U_{\alpha \beta}$ and $U_{\alpha \beta \gamma}$ intersections, ${ }^{6}$ one has

$$
\omega^{2}=d A_{\alpha}^{1}, \quad-A_{\alpha}^{1}+A_{\beta}^{1}=d a_{\alpha \beta}^{0}, \quad-a_{\alpha \beta}^{0}-a_{\beta \gamma}^{0}-a_{\gamma \alpha}^{0}=2 \pi n_{\alpha \beta \gamma} .
$$

The Kaluza-Klein space $C_{M}^{\left[\omega^{2}\right]}$ is constructed from $M$ by introducing a new coordinate $\tau_{\alpha}$ at each open set $U_{\alpha}$ with patching conditions

$$
\left(-\tau_{\alpha}+\tau_{\beta}-a_{\alpha \beta}^{0}\right) \quad \bmod 2 \pi \mathbb{Z}=0,
$$

which is consistent at triple overlaps $U_{\alpha \beta \gamma}$ if and only if $n_{\alpha \beta \gamma} \in \mathbb{Z}$ and so $\frac{1}{2 \pi}\left[\omega^{2}\right] \in H^{2}(M, \mathbb{Z})$. Taking the exterior derivative of patching condition, one finds that $d \tau_{\alpha}-A_{\alpha}^{1}=d \tau_{\beta}-A_{\beta}^{1}$ and so $d \tau-A^{1}$ is globally defined on the total space $C_{M}^{\left[\omega^{2}\right]}$. Thus $\pi^{*} \omega^{2}=-d\left(d \tau-A^{1}\right)$ is an exact form on $C_{M}^{\left[\omega^{2}\right]}$, and so $C_{M}^{\left[\omega^{2}\right]}$ satisfies the topological geometrization condition. Of course $C_{M}^{\left[\omega^{2}\right]}$ is a circle bundle on $M$ with first Chern class given by $\frac{1}{2 \pi}\left[\omega^{2}\right]$.

\footnotetext{
${ }^{5}$ Good covers exist for compact and non-compact manifolds and are essential in Čech- de Rham theory.

${ }^{6}$ We use the notation $U_{\alpha_{0} \ldots \alpha_{k}}=U_{\alpha_{0}} \cap \cdots \cap U_{\alpha_{k}}$ for the k-fold intersections or overlaps of open sets.
} 


\subsection{Patching C-spaces for closed 3-forms}

To begin the construction of $C_{M}^{\left[\omega^{3}\right]}$ spaces, suppose $M$ be a manifold and $\omega^{3}$ be a closed 3 -form on $M$. For applications in DFT, $M$ is the spacetime and $\omega^{3}$ is the NS-NS 3 -form field strength. In addition let $\left\{U_{\alpha}\right\}_{\alpha \in I}$ be a good cover of $M$ as for 2-forms in the previous section. Applying the Poincaré lemma on the open sets $U_{\alpha}$ as well double, triple and 4 -fold intersections, one finds that

$$
\begin{aligned}
\omega_{\alpha}^{3}=d B_{\alpha}^{2}, \quad-B_{\alpha}^{2}+B_{\beta}^{2} & =d a_{\alpha \beta}^{1}, \quad-a_{\alpha \beta}^{1}-a_{\beta \gamma}^{1}-a_{\gamma \alpha}^{1}=d a_{\alpha \beta \gamma}^{0}, \\
-a_{\beta \gamma \delta}^{0}+a_{\alpha \gamma \delta}^{0}-a_{\alpha \beta \delta}^{0}+a_{\alpha \beta \gamma}^{0} & =2 \pi n_{\alpha \beta \gamma \delta},
\end{aligned}
$$

respectively, where $n_{\alpha \beta \gamma \delta}$ are constants and the combinatorics of the open set labels follow from the definition of the Čech differential, see (6.1). $B_{\alpha}$ are the 2-form gauge potentials of $\omega^{3}$ on each $U_{\alpha}$, and $\left\{a_{\alpha \beta}^{1}, a_{\alpha \beta \gamma}^{0}\right\}$ are the patching or transition "functions" of $\omega^{3}$ at double and triple overlaps. Moreover if $\frac{1}{2 \pi} \omega^{3}$ represents a class in $H^{3}(M, \mathbb{Z})$, then $n_{\alpha \beta \gamma \delta} \in \mathbb{Z}$ on all 4 -fold overlaps, $U_{\alpha \beta \gamma \delta}$. All the patching data are skew-symmetric under the exchange of open set labels, ie $a_{\alpha \beta}^{1}=-a_{\beta \alpha}^{1}$ and similarly for the rest.

The gauge potentials $B_{\alpha}$ and the transition functions $\left\{a_{\alpha \beta}^{1}, a_{\alpha \beta \gamma}^{0}\right\}$ are not uniquely defined. In fact, the gauge potentials are defined up to the gauge transformations

$$
B_{\alpha}^{\prime}=B_{\alpha}+d \zeta_{\alpha}^{1}
$$

and similarly the transition functions are defined up to gauge transformations as

$$
\begin{aligned}
a_{\alpha \beta}^{\prime 1} & =a_{\alpha \beta}^{1}-\zeta_{\alpha}^{1}+\zeta_{\beta}^{1}+d \zeta_{\alpha \beta}^{0}, \\
a_{\alpha \beta \gamma}^{\prime 0} & =a_{\alpha \beta \gamma}^{0}-\zeta_{\alpha \beta}^{0}-\zeta_{\beta \gamma}^{0}-\zeta_{\gamma \alpha}^{0} .
\end{aligned}
$$

These gauge transformations are the only ones compatible with the closure of $\omega^{3}$.

The construction of $C_{M}^{\left[\omega^{3}\right]}$ proceeds with the introduction of new coordinates $y_{\alpha}^{1}$ and $\theta_{\alpha \beta}$ associated with the open sets $U_{\alpha}$ and the double overlaps $U_{\alpha \beta}$, respectively. These are new coordinates in addition to those of the spacetime. They should be thought in the same way as the Kaluza-Klein coordinate $\tau$ that we have introduced for the description of $C_{M}^{\left[\omega^{2}\right]}$ in the previous section. Though $y^{1}$ is assigned the degree of a 1-form. In addition, one imposes the patching conditions

$$
\begin{aligned}
-y_{\alpha}^{1}+y_{\beta}^{1}+d \theta_{\alpha \beta} & =a_{\alpha \beta}^{1}, \\
\left(\theta_{\alpha \beta}+\theta_{\beta \gamma}+\theta_{\gamma \alpha}+a_{\alpha \beta \gamma}^{0}\right) & =0 \bmod 2 \pi \mathbb{Z},
\end{aligned}
$$

on $U_{\alpha \beta}$ and $U_{\alpha \beta \gamma}$.

Using the second condition in (2.3), one finds that consistency of the first condition on triple overlaps yields

$$
d\left(\theta_{\alpha \beta}+\theta_{\beta \gamma}+\theta_{\gamma \alpha}+a_{\alpha \beta \gamma}^{0}\right)=0 .
$$

This is implied from the second condition in (2.6). Next investigating the consistency of the second condition of (2.6) on 4-fold overlaps and after using the last condition in (2.3), one finds that

$$
n_{\alpha \beta \gamma \delta}=0 \quad \bmod \mathbb{Z} .
$$

This is satisfied provided that $\frac{1}{2 \pi} \omega^{3}$ represents a class in $H^{3}(M, \mathbb{Z})$. 
One of the questions that arises in imposing (2.6) is how one is supposed to think about these new coordinates and their patching conditions. The coordinates should be thought in the same way as in the usual construction of a circle bundle over a manifold utilizing the patching conditions of a manifold together with those of a closed 2-form. For the gluing transformations, this particularly applies to the second patching condition which involves triple overlaps and three coordinates rather than double overlaps and two coordinates which appear in the usual patching for manifolds. To give some insight into this question, one can view the usual patching of manifolds as follows. Given two charts, ie open sets and coordinates adapted to each one of the sets, the patching condition at the double intersection relates the coordinates of first chart to the coordinates of the second chart, and vice versa. In this context, the second patching condition in (2.6) specifies how the three $\theta$ coordinates, each one associated with one of the three double overlaps that contribute to the triple overlap, are related.

\subsection{The $y^{1}$ coordinates}

To get some insight into the nature of $y$ coordinates, observe that the first patching condition in (2.6) can be solved to express the $y^{1}$ coordinates in terms of the angular coordinates $\theta$ using a partition of unity $\left\{\rho_{\alpha}\right\}_{\alpha \in I}$ subordinate to $\left\{U_{\alpha}\right\}_{\alpha \in I}$; for the definition of partitions of unity see eg [46] page 21. In particular, one has that

$$
y_{\alpha}^{1}=\tilde{y}_{\alpha}^{1}+\sum_{\gamma} \rho_{\gamma}\left(d \theta_{\alpha \gamma}-a_{\alpha \gamma}^{1}\right)
$$

where $\tilde{y}_{\alpha}^{1}$ are coordinates which transform as 1 -forms on $M, \tilde{y}_{\alpha}^{1}=\tilde{y}_{\beta}^{1}$. So $y_{\alpha}^{1}$ are coordinates which transform as 1 -forms of $M$ on $U_{\alpha}$ and receive an additional correction from the angular coordinates $\theta_{\alpha \gamma}$ and the transition functions $a_{\alpha \gamma}^{1}$ when they approach the double overlaps $U_{\alpha \gamma}$.

One of the consequences of $(2.9)$ is that the C-spaces $C_{M}^{\left[\omega^{3}\right]}$ are not manifolds. To see this, first observe that by construction there is a projection $\pi: \quad C_{M}^{\left[\omega^{3}\right]} \rightarrow M$. The dimension of the inverse image $\pi^{-1}(x)$ of $x \in M$ depends on $x$. If $x \in U_{\alpha}$ and $x \notin U_{\alpha_{0} \ldots \alpha_{k}}$, $\pi^{-1}(x)=\mathbb{R}^{n}$. While if $x \in U_{\alpha \beta}$ and $x \notin U_{\alpha \beta \gamma}$, then $\pi^{-1}(x)=\mathbb{R}^{n} \times S^{1}$. Finally if $x \in U_{\alpha \beta \gamma}$, then $\pi^{-1}(x)=\mathbb{R}^{n} \times T^{2}$, and so on. As a consequence $C_{M}^{\left[\omega^{3}\right]}$ may not have a well-defined dimension.

\subsection{Dependence on $\omega^{3}$}

Here we shall investigate whether or not $C_{M}^{\left[\omega^{3}\right]}$ depends on the representative $\omega^{3}$ of the class $\frac{1}{2 \pi}\left[\omega^{3}\right] \in H^{3}(M, \mathbb{Z})$. Suppose that $\omega^{\prime 3}$ is another representative of $\left[\omega^{3}\right]$, ie $\left[\omega^{\prime 3}\right]=\left[\omega^{3}\right]$. Then there is a globally defined 2-form $u^{2}$ such that $\omega^{\prime 3}=\omega^{3}+d u^{2}$. Thus $B_{\alpha}^{\prime 2}=B_{\alpha}^{2}+u_{\alpha}^{2}$. Since $u_{\alpha}^{2}=u_{\beta}^{2}$ at double overlaps the dependence on $u$ drops out and so $a_{\alpha \beta}^{1}$ does not dependent on the choice of representative of $\left[\omega^{3}\right]$. As a consequence the transition functions of $C_{M}^{\left[\omega^{3}\right]}$ do not depend on the representative of $\left[\omega^{3}\right]$.

There is additional gauge redundancy in the definition of $B_{\alpha}$ and that of the transition functions given in (2.4) and (2.5), respectively. This is eliminated by performing the 
compensating transformations

$$
y_{\alpha}^{\prime 1}=y_{\alpha}^{1}+\zeta_{\alpha}^{1}, \quad \theta_{\alpha \beta}^{\prime}=\theta_{\alpha \beta}+\zeta_{\alpha \beta}^{0},
$$

on the new coordinates. As a result $C_{M}^{\left[\omega^{3}\right]}$ does not depend on the choices made including that of the representative of $\left[\omega^{3}\right]$.

\subsection{Dependence on the cover}

One should also investigate a more subtle choice in the construction of $C_{M}^{\left[\omega^{3}\right]}$ that of the good cover $\left\{U_{\alpha}\right\}_{\alpha \in I}$. For this one can adapt a similar strategy as the one for manifolds which one starts from an atlas and adds all the compatible charts, ie all the charts which have smooth transition functions for each intersection amongst themselves and each intersection with the charts of the original atlas. Such a construction leads to the notion of the maximal atlas which characterizes the smooth structure on a manifold. Therefore if one begins with a topological space that can be given a manifold structure with respect to two different atlases which however lead to the same maximal atlas, then the two original spaces are identified as manifolds, ie the two original atlases give rise to the same smooth structure on the topological space. There is not necessarily a unique maximal atlas on a manifold as it is known that on a given topological manifold there can be more than one smooth structures.

In the same way one can add to a good cover $\left\{U_{\alpha}\right\}_{\alpha \in I}$ all the additional open sets (charts) which are compatible with the smooth structure of $M$ and give rise to a new maximal good cover on $M$. Then with respect to this maximal good cover one can define $C_{M}^{\left[\omega^{3}\right]}$. Moreover, one can assert that if two good covers give rise to the same maximal good cover, then the two original C-spaces must be identified. It is not apparent how the C-spaces with respect to two different maximal good covers are related. There can be a moduli of possibilities but this will not be unusual as many constructions and structures on spaces depend on the choice of open covers and atlases. ${ }^{7}$ Moreover the existence of a moduli will not invalidate the construction as each space provides a solution to the patching problem which has been the main question that has been addressed in this paper. It will simply mean that we have more than one solutions and the implications of this will be of interest to investigate. Furthermore, there is a mild indication that all such C-spaces have the same homotopy type as all of them have the effect to trivialize the class of $\omega^{3}$ and cohomology groups are homotopic invariant.

\subsection{Topological geometrization condition}

It has been argued in [40] that any space which geometrizes a k-form flux must be a Cspace, ie it admits a projection onto the spacetime such that the pull back of the k-form flux represents the trivial cohomological class in the C-space.

\footnotetext{
${ }^{7}$ The notion of the smooth structure is indeed atlas dependent. For example if one considers a triangle and takes the atlas induced on it as a $\mathbb{R}^{2}$ subspace, then the triangle is not a manifold because of the cusp singularities. However, the triangle is homeomorphic to a circle and so there is another atlas on the triangle inducing on it a smooth structure. The new atlas can be constructed explicitly.
} 
Here we shall demonstrate that $C_{M}^{\left[\omega^{3}\right]}$ is a C-space. As we have mentioned, there is a projection $\pi$ from $C_{M}^{\left[\omega^{3}\right]}$ onto $M$. Next taking the differential of the first patching condition in (2.6), one finds that

$$
-d y_{\alpha}^{1}+d y_{\beta}^{1}=d a_{\alpha \beta}^{1} .
$$

Using the second condition in (2.3), this can be rewritten as

$$
d y_{\alpha}^{1}-B_{\alpha}^{2}=d y_{\beta}^{1}-B_{\beta}^{2}
$$

Therefore $d y^{1}-B^{2}$ is globally defined on $C_{M}^{\left[\omega^{3}\right]}$. As $\pi^{*} \omega^{3}=-d\left(d y^{1}-B^{2}\right), \pi^{*} \omega^{3}$ is exact on $C_{M}^{\left[\omega^{3}\right]}$. Therefore $C_{M}^{\left[\omega^{3}\right]}$ satisfies the topological geometrization property.

\section{Relation to gerbes and generalized geometry}

\subsection{Gerbes}

In the definition of [41], a gerbe is the object which represents a class in $H^{3}(M, \mathbb{Z})$ in the same way that a circle bundle represents a class in $H^{2}(M, \mathbb{Z})$. It is expected that given a manifold $M$ and a class in $H^{3}(M, \mathbb{Z})$, in a certain sense, the gerbe is uniquely specified. In a direct analogy with circle bundles, gerbes are investigated via their transition functions. To relate the transition functions of a gerbe as defined in [41] to the transition functions we use here for $C_{M}^{\left[\omega^{3}\right]}$, write

$$
g_{\alpha \beta \gamma}=e^{i a_{\alpha \beta \gamma}^{0}} .
$$

Then the second equation in $(2.3)$ reads as

$$
g_{\beta \gamma \delta}^{-1} g_{\alpha \gamma \delta} g_{\alpha \beta \delta}^{-1} g_{\alpha \beta \gamma}=1,
$$

which can be recognized as the patching condition of a gerbe on a 4-fold overlap.

Therefore $C_{M}^{\left[\omega^{3}\right]}$ is a gerbe. But the emphasis in the construction of $C_{M}^{\left[\omega^{3}\right]}$ is different. Instead of focusing on the transition functions, $C_{M}^{\left[\omega^{3}\right]}$ describes the object itself. Furthermore $C_{M}^{\left[\omega^{3}\right]}$ is possibly one of the many representatives of $\left[\omega^{3}\right] \in H^{3}(M, \mathbb{Z})$ that has been chosen such that it can apply to DFT. In fact, this is the case even for the elements of $H^{2}(M, \mathbb{Z})$. To see this note that these can be represented with complex line bundles $L$ as well. Furthermore $L$ and the direct sum $L \oplus I$, where $I$ is the trivial $I$ line bundle, represent the same class in $H^{2}(M, \mathbb{Z})$. Clearly $L$ and $L \oplus I$ have different geometric properties which can be essential in certain applications.

The construction of $C_{M}^{\left[\omega^{3}\right]}$ via the introduction of $y^{1}$ and $\theta$ coordinates at the open sets and double overlaps and their patching according to (2.3) are essential for the applications considered here. Note for example that for the description of the gerbe patching data $g_{\alpha \beta \gamma}$, these coordinates are not necessary. Presumably there are other spaces with different geometric properties from $C_{M}^{\left[\omega^{3}\right]}$ that represent the same class in $H^{3}(M, \mathbb{Z})$. 


\subsection{Generalized geometry}

\subsubsection{Closed 2-forms}

Before we investigate the relation between $C_{M}^{\left[\omega^{3}\right]}$ spaces and generalized geometry [43-45], it is instructive to examine how the geometry of the spacetime is related to that of the Kaluza-Klein space $C_{M}^{\left[\omega^{2}\right]}$. On the spacetime, one can define an extension $E$ of the tangent bundle $T M$ with a trivial bundle $I, 0 \rightarrow I \stackrel{i}{\rightarrow} E \stackrel{j}{\rightarrow} T M \rightarrow 0$ which has transition functions

$$
X_{\alpha}=X_{\beta}, \quad f_{\alpha}=f_{\beta}-X_{\beta}\left(a_{\alpha \beta}^{0}\right),
$$

where $a_{\alpha \beta}^{0}$ are the transition functions of the 2 -form $\omega^{2}$.

Choosing a splitting $h: T M \rightarrow E$, one can define the twisted bracket given by

$$
[h(X)+i(f), h(Y)+i(g)]_{\omega^{2}}=h([X, Y])+i\left(X(g)-Y(f)+\omega^{2}(X, Y)\right) .
$$

This bracket by construction is preserved by the patching conditions (3.3).

To give a geometric interpretation to the construction above, observe that $T C_{M}^{\left[\omega^{2}\right]}$ is also an extension of $\pi^{*} T M$ with a trivial bundle $I$; at every point $p \in C_{M}^{\left[\omega^{2}\right]}, T C_{M}^{\left[\omega^{2}\right]}$ has a preferred direction that of the the tangent bundle of the fibre $S^{1}$. Furthermore $C_{M}^{\left[\omega^{2}\right]}$ is equipped with a globally defined 1-form $d \tau-A$, which is a principal bundle connection, and so splits $T C_{M}^{\left[\omega^{2}\right]}$ into horizontal and vertical subspaces. In particular the horizontal lift of a vector field $X$ on $T M$ to $T C_{M}^{\left[\omega^{2}\right]}$ is

$$
X^{h}=X^{i}\left(\frac{\partial}{\partial x^{i}}+A_{i} \frac{\partial}{\partial \tau}\right) .
$$

Then observe that the Lie bracket of the $S^{1}$-invariant sections of $T C_{M}^{\left[\omega^{2}\right]}$ which can be written as $X^{h}+f \frac{\partial}{\partial \tau}$ is

$$
\left[X^{h}+f \frac{\partial}{\partial \tau}, Y^{h}+g \frac{\partial}{\partial \tau}\right]=[X, Y]^{h}+\left(X(g)-Y(f)+\omega^{2}(X, Y)\right) \frac{\partial}{\partial \tau} .
$$

Therefore, the Lie bracket reproduces the twisted bracket (3.4) upon setting $h(X)=X^{h}$ and $i(f)=f \frac{\partial}{\partial \tau}$. From the physics point of view, the bracket (3.6) arises in the quantization of a charged particle in a magnetic field carrying Kaluza-Klein momentum in the extra direction $\tau$.

\subsubsection{Closed 3-forms}

Generalized geometry [43-45] on $M$ is based on the extension $E$ of the tangent bundle $T M, 0 \rightarrow T^{*} M \stackrel{i}{\rightarrow} E \stackrel{j}{\rightarrow} T M \rightarrow 0$. The patching conditions of $E$ are

$$
X_{\alpha}=X_{\beta}, \quad \zeta_{\alpha}=\zeta_{\beta}-\iota_{X_{\beta}} d a_{\alpha \beta}^{1},
$$

where $d a_{\alpha \beta}^{1}$ satisfies the Čech co-cycle condition, $d a_{\alpha \beta}^{1}+d a_{\beta \gamma}^{1}+d a_{\gamma \alpha}^{1}=0$, as it can be seen from (2.3). Choosing a splitting $h: T M \rightarrow E$, one can define a twisted Courant bracket on $E$ given by

$$
[h(X)+i(\zeta), h(Y)+i(\eta)]_{\omega^{3}}^{C}=h([X, Y])+i\left(\mathcal{L}_{X} \eta-\mathcal{L}_{Y} \zeta-\frac{1}{2} d(\eta(X)-\zeta(Y))-\iota_{X} \iota_{Y} \omega^{3}\right) .
$$


This bracket by construction is well-defined. For use later, the untwisted Courant bracket $[X+\zeta, Y+\eta]^{C}$, where $X, Y$ are vector fields and $\zeta, \eta$ are 1 -forms, is defined as above after suppressing the maps $i, h$ and removing the term $\iota_{X} \iota_{Y} \omega^{3}$.

To give a geometric interpretation to the above construction, let us view it from the perspective of $C_{M}^{\left[\omega^{3}\right]}$. The tangent bundle of $C_{M}^{\left[\omega^{3}\right]}$ is not well-defined. However, we can define a bundle $\mathcal{E}$ spanned by $\left(\frac{\partial}{\partial x^{i}}, \frac{\partial}{\partial y_{j}}\right)$, where we note from (2.9) that $d y=d y_{i} \wedge d x^{i}$ and $\frac{\partial}{\partial y_{j}}$ is defined as the dual of $d y_{i}$, ie $\left\langle d y_{i}, \frac{\partial}{\partial y_{j}}\right\rangle=\delta^{j}$. We have suppressed the degree of $y$ as well as the open set labeling. This definition is in direct analogy to that of $T C_{M}^{\left[\omega^{2}\right]}$ for which the fibres are spanned by $\left(\frac{\partial}{\partial x^{i}}, \frac{\partial}{\partial \tau}\right)$, and the observation that the patching conditions

$$
-d y_{\alpha}^{1}+d y_{\beta}^{1}=d a_{\alpha \beta}^{1},
$$

do not depend on the $\theta$ coordinates. It is clear from the patching conditions that $\mathcal{E}$ is an extension $0 \rightarrow \pi^{*} T^{*} M \stackrel{k}{\rightarrow} \mathcal{E} \stackrel{\ell}{\rightarrow} \pi^{*} T M \rightarrow 0$.

To continue observe that $d y-B$ defines a map from $\pi^{*} T M$ into $\mathcal{E}^{*}$. Therefore its dual $(d y-B)^{*}$ defines a map from $\mathcal{E}$ to $\pi^{*} T^{*} M$ and this is a analogous structure to a principal bundle connection. So $\mathcal{E}$ can be split into horizontal and vertical subspaces. In particular the horizontal lift of a vector field $X$ on $M$ is

$$
X^{h}=X^{i}\left(\frac{\partial}{\partial x^{i}}+B_{i j} \frac{\partial}{\partial y_{j}}\right)
$$

while the vertical subspace is spanned by $\left(\frac{\partial}{\partial y_{j}}\right)$. The sections of $\mathcal{E}$ which depend only on the coordinates of $M$ can be written as $X^{h}+k(\zeta)$, where

$$
k(\zeta)=\zeta_{i} \frac{\partial}{\partial y_{i}} .
$$

Then observe that the computation of the (untwisted) Courant bracket gives

$$
\left[X^{h}+k(\zeta), Y^{h}+k(\eta)\right]^{C}=[X, Y]^{h}+k\left(\mathcal{L}_{X} \eta-\mathcal{L}_{Y} \zeta-\frac{1}{2} d(\eta(X)-\zeta(Y))-\iota_{X} \iota_{Y} \omega^{3}\right) .
$$

The right hand side gives the twisted Courant bracket (3.8) upon setting $h(X)=X^{h}$ and $i(\zeta)=\zeta_{i} \frac{\partial}{\partial y_{i}}$. Observe that, unlike the naive Lie bracket on $\mathcal{E}$, the Courant bracket transforms covariantly under shifts related to the choice of a representative for $B$. This can readily be seen from the expression above.

We can also globally define tensors on $C_{M}^{\left[\omega^{3}\right]}$, like for example a generalized metric

$$
G=g_{i j} d x^{i} d x^{j}+g^{i j}\left(d y_{i}+B_{i k} d x^{k}\right)\left(d y_{j}+B_{j \ell} d x^{\ell}\right),
$$

where $g$ is a metric on $M$.

\section{Some topological aspects of $C_{M}^{\left[\omega^{3}\right]}$ and an example}

\subsection{Topological aspects}

One way to get an insight into the topological structure of a C-space it is instructive to investigate $C_{M}^{\left[\omega^{3}\right]}$ in a chain complex approximation of the spacetime. Given a good cover 
$\left\{U_{\alpha}\right\}_{\alpha \in I}$ on $M$, one can associate a chain complex with $M$ the nerve $\mathcal{N}$ of the cover, see eg [46] page 100. $\mathcal{N}$ is constructed as follows. One introduces a vertex for each open set $U_{\alpha}$ of the cover. Two vertices are joined by an edge if and only if the corresponding open sets intersect $U_{\alpha \beta} \neq \emptyset$. The faces of three edges of a triangle are filled if and only if the corresponding three open sets have a common intersection $U_{\alpha \beta \gamma} \neq \emptyset$, and so on. The cohomology of this chain complex is exactly the same as the de Rham cohomology or singular cohomology depending on the coefficients.

Let us now focus how the information from the additional coordinates of $C_{M}^{\left[\omega^{3}\right]}$ can be stored on the nerve $\mathcal{N}$. This particularly applies to the angular coordinates $\theta_{\alpha \beta}$ as the $y_{\alpha}^{1}$ coordinates are contractible. It is apparent from the construction of $C_{M}^{\left[\omega^{3}\right]}$ that the vertices of $\mathcal{N}$ do not alter as there are no angular coordinates associated to open sets. However a circle is associated to every point of an edge in $\mathcal{N}$ as these represent the intersection of two open sets. Furthermore at every point on a face of $\mathcal{N}$ one should associate a 2 -torus. This is because of the second patching condition in (2.6) as the three angular coordinates associated to each edge are restricted to two.

Therefore one can describe this construction at a face of $\mathcal{N}$ as follows. The 2-torus of the face degenerates to circles at each of the three edges, and in turn, the circles at the edges and the tori of the face degenerate to a point as they approach the vertices. Such a structure is reminiscent ${ }^{8}$ to that of $\mathbb{C} P^{2}$. To see this consider the algebraic equation of $S^{5}$,

$$
w_{1} \bar{w}_{1}+w_{2} \bar{w}_{2}+w_{3} \bar{w}_{3}=1 .
$$

Setting $t_{1}=w_{1} \bar{w}_{1}, t_{2}=w_{2} \bar{w}_{2}$ and $t_{3}=w_{3} \bar{w}_{3}$, this can be seen as the defining equation of a 2-simplex. The three phases of the complex numbers $w_{1}, w_{2}$ and $w_{3}$ associate a circle at every vertex, a 2 -torus at every point of a edge, and a 3 -torus at every point of the face. As $\mathbb{C} P^{2}$ is the base space of the fibration, $S^{1} \rightarrow S^{5} \rightarrow \mathbb{C} P^{2}$, where $S^{1}$ acts from the right on the triplet $\left(w_{1}, w_{2}, w_{3}\right)$, a circle is removed from every point of the simplex leading to the picture describe above for $\mathcal{N}$. If such a topology is put on $C_{M}^{\left[\omega^{3}\right]}$, it would be different from that of spacetime $M$. As we shall see $\mathbb{C} P^{2}$, or rather $\mathbb{C} P^{\infty}$, appears also in the homotopy approach to C-spaces using Whitehead towers.

\subsection{The C-space of 3-torus with a 3 -form flux}

The construction of $C_{M}^{\left[\omega^{3}\right]}$ described in section 2 is general and applies to every manifold with a good cover equipped with a closed 3 -form which represents a class in $H^{3}(M, \mathbb{Z})$. As good covers exist on manifolds, one can construct $C_{M}^{\left[\omega^{3}\right]}$ for all smooth solutions of supergravity theories including that of the NS5-brane. ${ }^{9}$

Here we construct the C-space of a 3-torus with a 3-form flux. This example was initially investigated from the perspective of double spaces in [33]. Later it was explored from the patching point of view in [40] where it was found that the construction depends on the choice of the atlas on $T^{3}$. Another feature of the construction was that a quantization

\footnotetext{
${ }^{8}$ This construction has been adapted to construct the universal bundle classifying spaces for any group, see eg [47].

${ }^{9}$ The dilaton singularity does not affect the construction.
} 
condition was imposed at the triple overlaps rather than the 4-fold overlaps as required by the Dirac quantization condition of 3-forms field strengths.

We shall follow the notation of [40] where all the data regarding the patching conditions of the 3 -form flux can be found. ${ }^{10}$ The patching conditions of the C-space are

$$
\begin{aligned}
-y_{\alpha_{1}}^{1}+y_{\alpha_{2}}^{1}+d \theta_{\alpha \beta} & =a_{\alpha_{1} \alpha_{2}}^{1}, \\
\left(\theta_{\alpha_{1} \alpha_{2}}+\theta_{\alpha_{2} \alpha_{3}}+\theta_{\alpha_{3} \alpha_{1}}+a_{\alpha_{1} \alpha_{2} \alpha_{3}}^{0}\right) & =0 \bmod 2 \pi \mathbb{Z},
\end{aligned}
$$

where we have set $\alpha_{1}=i_{1} j_{1} k_{1}$ and so on. In the atlas we have chosen on $T^{3}$, the components of $a_{\alpha_{1} \alpha_{2}}^{1}$ and $a_{\alpha_{1} \alpha_{2} \alpha_{3}}^{0}$ are linear in the coordinates of $T^{3}$. However the above patching conditions do not depend on this choice. This particularly applies to the second condition in (4.2) as the consistency required for it leads to $n_{\alpha_{1} \alpha_{2} \alpha_{3} \alpha_{4}} \in \mathbb{Z}$ on 4 -fold overlaps. Since $n_{\alpha_{1} \alpha_{2} \alpha_{3} \alpha_{4}}$ are constant for any choice of an atlas, the quantization condition is atlas independent. This should be contrasted with the DFT calculation which for consistency requires that the components of the 1-form $d a_{\alpha_{1} \alpha_{2} \alpha_{3}}^{0}$ should be constant and that they should identified periodically up to some period. As $d a_{\alpha_{1} \alpha_{2} \alpha_{3}}^{0}$ is a local 1-form, the constancy of its components is an atlas dependent statement [40].

\section{DFT on double manifolds}

\subsection{Revisiting the patching of double manifolds}

In the formulation of DFT so far, one introduces a new set of coordinates ${ }^{11} \tilde{x}$ in addition to those of the spacetime $x$ and imposes on all the fields and their transformations the strong section condition which reads

$$
\frac{\partial}{\partial x^{i}} F \frac{\partial}{\partial \tilde{x}_{i}} G+\frac{\partial}{\partial x^{i}} G \frac{\partial}{\partial \tilde{x}_{i}} F=0, \quad \frac{\partial}{\partial x^{i}} \frac{\partial}{\partial \tilde{x}_{i}} F=0 .
$$

Setting for $F$ and $G$ the infinitesimal local transformations $\delta x^{i}$ and $\delta \tilde{x}_{i}$ of $x^{i}$ and $\tilde{x}_{i}$, respectively, and assuming that $\delta x^{i}$ must be arbitrary functions of $x$, which is required in order to account for all reparameterizations the spacetime, ${ }^{12}$ one concludes that the most general solutions to the above conditions are

$$
\delta x^{i}=\xi^{i}(x), \quad \delta \tilde{x}_{i}=\kappa_{i}(x) .
$$

In particular, the first equation in (5.1) for $F=G=\delta x^{i}$ implies that $\delta x^{i}$ can depend only on $x$. Then again the first equation for $F=\delta x^{i}$ and $G=\delta \tilde{x}_{i}$ implies that $\delta \tilde{x}_{i}$ can dependent only on $x$ as well. These infinitesimal transformations can be integrated to give

$$
x^{\prime i}=x^{\prime i}\left(x^{j}\right), \quad \tilde{x}_{i}^{\prime}=\tilde{x}_{i}-\kappa_{i}(x) .
$$

\footnotetext{
${ }^{10}$ Strictly speaking one should introduce a third open set on $S^{1}, U_{3}=\left(-\frac{\pi}{4}, \frac{\pi}{4}\right)$, so that the cover is a good cover. As the transition functions between $U_{1}$ and $U_{3}$, and $U_{2}$ and $U_{3}$ are the identity, there is no change in the computations on [40] and the effects of $U_{3}$ have already been taken into account via the choice of $n_{x}$.

${ }^{11}$ In [40] the dual coordinates were denoted with $y$. Here we denote them with $\tilde{x}$ to distinguish them from those of the C-spaces as they have different transformation properties.

${ }^{12}$ It is required for example for the construction of a maximal atlas on the spacetime or equivalently general covariance.
} 
Moreover in $[33,34], \kappa$ is related linearly to the gauge transformations of the $B$ field. To investigate the global properties of DFTs, these transformations are interpreted as patching conditions,

$$
x_{\alpha}^{i}=x_{\alpha \beta}^{i}\left(x_{\beta}\right), \quad \tilde{x}_{\alpha}=\tilde{x}_{\beta}-\kappa_{\alpha \beta}\left(x_{\beta}\right),
$$

where we have introduced a good cover $\left\{U_{\alpha}\right\}_{\alpha \in I}$ on the spacetime $M$.

The strong section condition has another solution where $z$ and $x$ exchange places, this is the solution for the dual space. It also has many more solutions ${ }^{13}$ provided that one weakens the requirement that $\delta x^{i}$ must be an arbitrary function of $x$ and does not allow for general reparametrizations of $M$. But this breaks general covariance.

So in order to allow for spacetime reparametrization invariance, one is forced to patch the theory with transformations of the type (5.4). If this is the case, then

$$
\kappa_{\alpha \beta}+\kappa_{\beta \gamma}+\kappa_{\gamma \alpha}=0 .
$$

Using the results of [40], one concludes that this is possible if and only if the double space is diffeomorphic to $D_{M}=T^{*} M$. This is because the condition (5.5) implies that $\kappa_{\alpha \beta}=-\zeta_{\alpha}+\zeta_{\beta}$ and so after a redefinition of the $\tilde{x}$ coordinates transform as 1-forms.

This result is independent from the form of finite transformations on the fields and other geometric considerations. It is a consequence of the application of the strong section condition. Thus if one uses the strong section condition to describe the double theory and allows for general reparameterizations of the spacetime coordinates, then one is led to the conclusion that the double space is $T^{*} M$.

This has immediate consequences. First if the transformations of the dual coordinates $\tilde{x}$ do not transform under the B-field gauge transformation, it appears to contradict standard T-duality results like that of the $S^{3}$ and $S^{3} / \mathbb{Z}_{p}$ pair. Consistency of the construction of this T-duality pair requires that the Hopf fibre coordinate $\tilde{\theta}$ of $S^{3} / \mathbb{Z}_{p}$, which can be identified as the dual coordinate of the fibre coordinate $\theta$ of $S^{3}$, transforms non-trivially under the B-field patching conditions of the $S^{3}$ solution. Thus the identification of the double space with $T^{*} M$ is in conflict with examples.

Furthermore, $T^{*} M$ is contractible to $M$, so $\pi^{*} \omega^{3}$ is not trivial in $T^{*} M$. Thus this space does not satisfy the topological geometrization condition. In addition, if the transition functions of $\omega^{3}$ at double overlaps are related via a linear transformations to $\kappa$, then $\omega^{3}$ is exact [40].

As a final remark, one can try to patch the double space using both spacetime and dual space patching conditions as

$$
\begin{array}{ll}
x_{\alpha}=x_{\alpha \beta}\left(x_{\beta}\right), & \tilde{x}_{\alpha}=\tilde{x}_{\beta}-\kappa_{\alpha \beta}\left(x_{\beta}\right), \\
\tilde{x}_{\gamma}=\tilde{x}_{\gamma \alpha}\left(\tilde{x}_{\alpha}\right), & x_{\gamma}=x_{\alpha}-\tilde{\kappa}_{\gamma \alpha}\left(\tilde{x}_{\alpha}\right)
\end{array}
$$

on $U_{\alpha \beta}$ and $U_{\gamma \alpha}$, respectively, and seek consistency at the triple overlap $U_{\alpha \beta \gamma}$. However, it is straightforward to see that the patching conditions on $U_{\beta \gamma}$ do not satisfy the strong section condition.

\footnotetext{
${ }^{13}$ One can easily construct many power series solutions.
} 


\subsection{Relation of double spaces to C-spaces}

Now let us compare the results of the previous section with those we have obtained for the $C_{M}^{\left[\omega^{3}\right]}$ spaces in section 2. In particular, let us compare the second patching condition of (5.4) with the first patching condition in (2.6). It is clear (2.6) reduces to (5.4) only when the new coordinate $\theta_{\alpha \beta}$ is chosen ${ }^{14}$ as

$$
\theta_{\alpha \beta}=0
$$

$y^{1}=\tilde{x}$ and $\kappa_{\alpha \beta}=a_{\alpha \beta}^{1}$. This choice cannot be made everywhere on $M$ consistent with the data. Thus the double spaces are local subspaces of $C_{M}^{\left[\omega^{3}\right]}$.

Although the geometric aspects of DFT on $C_{M}^{\left[\omega^{3}\right]}$ have not been developed, it is clear from the topological considerations presented that for the global definition of DFT additional coordinates are required. The mere introduction of $\tilde{x}$ coordinates in the context of double spaces is not sufficient to geometrize the topological charges of $\omega^{3}$, and to give a global definition of double spaces. The examination of the example of [33] from the patching point of view in [40] and in section 4.2 supports this assertion. However, it is not apparent how the additional coordinates $\theta$ can be inserted in the description of DFTs.

\section{$6 \quad$ C-spaces for closed k-forms}

\subsection{The construction of $C_{M}^{\left[\omega^{k}\right]}$}

The construction of C-spaces for $\omega^{k}$ closed forms, $C_{M}^{\left[\omega^{k}\right]}$, can be done in a way similar to that for $C_{M}^{\left[\omega^{3}\right]}$. To simplify the discussion it is convenient to introduce the Čech differential $\delta$. As before we choose a good cover $\left\{U_{\alpha}\right\}_{\alpha \in I}$ on $M$ and define

$$
\delta \lambda_{\alpha_{0} \alpha_{1} \ldots \alpha_{p}}^{m}=\sum_{i=0}^{p}(-1)^{i} \lambda_{\alpha_{0} \ldots \alpha_{i-1}}^{m} \hat{\alpha}_{i} \alpha_{i+1} \ldots \ldots \alpha_{p},
$$

where $\lambda^{m}$ is a m-form defined at p-overlaps and restricted upon applying $\delta$ to $(p+1)$ overlaps, and $\hat{\alpha}_{i}$ means that the label $\alpha_{i}$ is omitted. As before all these forms defined at the various overlaps are skew-symmetric under the exchange of the labels of the open sets. For example,

$$
\delta \lambda_{\alpha_{0} \alpha_{1}}^{m}=-\lambda_{\alpha_{0}}^{m}+\lambda_{\alpha_{1}}^{m},
$$

on $U_{\alpha_{0} \alpha_{1}}$. Observe that $\delta^{2}=0$ and $d \delta=\delta d$.

Applying the Poincaré lemma, the Čech-de Rham expansion of a k-form at multiple overlaps is

$$
\omega_{\alpha}^{k}=d A_{\alpha}^{k-1}, \quad \delta A_{\alpha_{0} \alpha_{1}}^{k-1}=d a_{\alpha_{0} \alpha_{1}}^{k-2}, \ldots, \delta a_{\alpha_{0} \ldots \alpha_{\ell}}^{k-\ell}=d a_{\alpha_{0} \ldots \alpha_{\ell}}^{k-\ell-1}, \ldots, \delta a_{\alpha_{0} \ldots \alpha_{k}}^{0}=2 \pi n_{\alpha_{0} \ldots \alpha_{k}},
$$

where $n_{\alpha_{0} \ldots \alpha_{k}}$ are constants. Again $\frac{1}{2 \pi} \omega^{k}$ represents a class in $H^{k}(M, \mathbb{Z})$, iff $n_{\alpha_{0} \ldots \alpha_{k}} \in \mathbb{Z}$.

\footnotetext{
${ }^{14}$ If $\theta_{\alpha \beta}$ was not identified $\bmod 2 \pi \mathbb{Z}$, it would have been sufficient to choose it as a function of $U_{\alpha \beta}$.
} 
The transition functions of the $\omega^{k}$ are not unique. Rather they are specified up to the gauge transformations

$$
a_{\alpha_{0} \ldots \alpha_{\ell+1}}^{k-\ell}=a_{\alpha_{0} \ldots \alpha_{\ell+1}}^{k-\ell}+d \zeta_{\alpha_{0} \ldots \alpha_{\ell+1}}^{k-\ell-1}+\delta \zeta_{\alpha_{0} \ldots \alpha_{\ell+1}}^{k-\ell} .
$$

To construct $C_{M}^{\left[\omega^{k}\right]}$ introduce coordinates $y_{\alpha_{0} \ldots \alpha_{\ell}}^{k-\ell}$ and impose the patching conditions

$$
\begin{aligned}
\delta y_{\alpha_{0} \ldots \alpha_{\ell+1}}^{k-\ell}+d y_{\alpha_{0} \ldots \alpha_{\ell+1}}^{k-\ell-1} & =a_{\alpha_{0} \ldots \alpha_{\ell+1}}^{k-\ell}, \quad \ell=2, \ldots, k-1, \\
\left(\delta y_{\alpha_{0} \ldots \alpha_{k}}^{0}-a_{\alpha_{0} \ldots \alpha_{k}}^{0}\right) & =0 \bmod 2 \pi \mathbb{Z},
\end{aligned}
$$

where now $y^{0}$ denote the new angular coordinates. After acting with $\delta$, it is clear from the last patching condition that consistency requires that $n_{\alpha_{0} \ldots \alpha_{k+1}} \in \mathbb{Z}$ and so $\frac{1}{2 \pi} \omega^{k}$ represents a class in $H^{k}(M, \mathbb{Z})$. This is the Dirac quantization condition. Note that the construction begins with the introduction of a new coordinate which locally is a $(k-2)$ form as expected from considerations that apply to exceptional field theories containing a $\mathrm{k}$-form. Then proceed with the introduction of many new other coordinates at the multiple overlaps of the open sets of the good cover.

The construction of $C_{M}^{\left[\omega^{k}\right]}$ is independent from the choice of the transition functions in (6.4) provided we allow the new coordinates to transform as

$$
y_{\alpha_{0} \ldots \alpha_{\ell}}^{\prime k-\ell}=y_{\alpha_{0} \ldots \alpha_{\ell}}^{k-\ell}+\zeta_{\alpha_{0} \ldots \alpha_{\ell}}^{k-\ell} .
$$

In addition one can show that $C_{M}^{\left[\omega^{k}\right]}$ depends only on the class of $\frac{1}{2 \pi} \omega^{k}$ in $H^{k}(M, \mathbb{Z})$. Furthermore, $C_{M}^{\left[\omega^{k}\right]}$ obeys the topological geometrization condition. In particular, it is easy to see from the construction above that $d y_{\alpha}^{k-2}-A_{\alpha}^{k-1}=d y_{\beta}^{k-2}-A_{\beta}^{k-1}$ and so $\pi^{*} \omega^{k}=$ $-d\left(d y^{k-2}-A^{k-1}\right)$ is exact on the C-space.

\subsection{Applications}

Most of the properties and applications we have explored for $C_{M}^{\left[\omega^{3}\right]}$ can be extended to $C_{M}^{\left[\omega^{k}\right]}$. Selectively, on $C_{M}^{\left[\omega^{k}\right]}$ one can introduce an extension

$$
0 \rightarrow \pi^{*} \Lambda^{k-2}(M) \rightarrow \mathcal{E} \rightarrow \pi^{*} T M \rightarrow 0
$$

where $\Lambda^{k-2}(M)$ is the bundle of (k-2)-forms. As $d y_{\alpha}^{k-2}-A_{\alpha}^{k-1}$ is globally defined on $C_{M}^{\left[\omega^{k}\right]}$ introduces a splitting of $\mathcal{E}$ and using this one can introduce a bracket and write a generalized metric in a way similar to that of $C_{M}^{\left[\omega^{3}\right]}$ presented in section 3.2. $C_{M}^{\left[\omega^{k}\right]}$ provides also a model for a k-gerbe.

In the context of exceptional field theories, the strong section condition, under similar assumptions to the DFT case, will lead to a patching condition

$$
-\tilde{x}_{\alpha}^{k-2}+\tilde{x}_{\beta}^{k-2}=\kappa_{\alpha \beta}^{k-2} .
$$

for the (k-2)-form coordinates. Again this implies that the exceptional spaces are diffeomorphic to $\Lambda^{k-2}(M)$. Such a space cannot satisfy the topological geometrization condition. Furthermore if $\kappa_{\alpha \beta}^{k-2}$ are related to the transition functions of $\omega^{k}$ at double overlaps with a 
linear map, then $\omega^{k}$ represents the trivial class in cohomology. The exceptional spaces are local subspaces of $C_{M}^{\left[\omega^{k}\right]}$ where all coordinates of the latter apart from $x$ and $y^{k-2}$ are set to zero. These topological considerations lead to the conclusion that for the global definition of exceptional field theories many more coordinates are needed in analogy with DFTs.

\section{Whitehead towers and $C_{M}^{\left[\omega^{k}\right]}$}

To get a new insight into C-spaces, it is helpful to find alternative constructions which are not based on local data. For this as a guidance, one can use the topological geometrization property. It has been mentioned in [40], that there is such a construction in homotopy theory realized by the Whitehead towers. As we shall see Whitehead towers include the Kaluza-Klein construction and provide a homotopy model for spaces that satisfy the topological geometrization condition. Moreover when applied to 3-forms have an intriguing connections to gerbes.

The Whitehead towers are sequences of fibrations such that

$$
M \stackrel{p_{1}}{\longleftarrow} X_{1} \stackrel{p_{2}}{\longleftarrow} X_{2} \stackrel{p_{3}}{\longleftarrow} X_{3} \stackrel{p_{4}}{\longleftarrow} \ldots
$$

where the fibre associated with the $p_{n}$ projection is the Eilenberg-MacLane space $K\left(n, \pi_{n-1}\right), \pi_{\ell}=\pi_{\ell}(M)$ are the homotopy groups of $M$, and $X_{n}$ is n-connected, ie $\pi_{\ell}\left(X_{n}\right)=0$ for $\ell \leq n$ and also $\pi_{\ell}\left(X_{n}\right)=\pi_{\ell}(M)$ for $\ell>n$. The Eilenberg-MacLane space $K(m, A)$ has the property that $\pi_{\ell}(K(m, A))=0$ unless $\ell=m$ in which case $\pi_{m}(K(m, A))=A$ for any abelian group $A$.

Assuming that $M$ is connected, the description of $X_{1}$ begins with the construction of an auxiliary space $Y_{1}$ which is derived from $M$ after adding cells to kill all the higher homotopy groups than $\pi_{1} . M$ is included in $Y_{1}$. Then a point $z$ is chosen in $Y_{1}$, and $X_{1}$ is defined as all paths that begin at $z$ and end in $M$ as $M \subset Y_{1}$. Then $p_{1}$ is defined as the end point projection of the paths. It turns out that the fibre of this fibration is homotopic to the loop space $\Omega_{*}\left(Y_{1}\right)$ which is the fibre over $z$. As by construction $Y_{1}=K\left(\pi_{1}, 1\right)$, one concludes from the homotopy exact sequence of path fibrations that $\Omega_{*}\left(Y_{1}\right)=K\left(\pi_{1}, 0\right)$. As the only non-vanishing homotopy group is $\pi_{0}\left(K\left(\pi_{1}, 0\right)\right)=\pi_{1}$, from the homotopy exact sequence of the fibration $M \stackrel{p_{1}}{\longleftarrow} X_{1}$ one finds that $X_{1}$ homotopic to the universal cover of $M$, ie $X_{1}$ is simply connected, and $\pi_{\ell}\left(X_{1}\right)=\pi_{\ell}(M)$ for all $\ell>1$. This construction can be repeated for $X_{1}$ to yield $X_{2}$ and so on, see eg [46] page 252 .

Next assume that $M$ is simply connected so that we can go straight to the fibration $M \stackrel{p_{2}}{\longleftarrow} X_{2}$. The fibre in this case is $K\left(\pi_{2}, 1\right)$ and $\pi_{2}=H_{2}(M, \mathbb{Z})=H^{2}(M, \mathbb{Z})$ as $M$ is simply connected. Since $\pi_{1}\left(X_{2}\right)=\pi_{2}\left(X_{2}\right)=0, H^{2}\left(X_{2}, \mathbb{Z}\right)=0$ and so $X_{2}$ realizes the topological geometrization property for $M$ and for all closed 2-forms on $M$. Furthermore, the construction is homotopic to the usual Kaluza-Klein reduction. This is because for $\pi_{2}=\oplus^{m} \mathbb{Z}$, the fibre $K\left(\oplus^{m} \mathbb{Z}, 1\right)$ can be chosen up to a homotopy as $T^{m}$. Though there is a difference between $X_{2}$ and $C_{M}^{\left[\omega^{2}\right]}$ as the former by construction topologically geometrizes all closed 2-forms while the latter topologically geometrizes only $\omega^{2}$. Of course one can repeat the process to construct the C-spaces for all the generators of closed 2-forms in which case both $C_{M}^{\left[\omega^{2}\right]}$ and $X_{2}$ will have the same homotopy groups. 
Next let us go one step up the Whitehead tower. Assume that $M$ is 2-connected. In such case, $X_{3}$ is 3-connected and realizes the topological geometrization property for $M$ and for all closed 3-forms on $M$. Furthermore, for $\pi_{3}=\oplus^{m} \mathbb{Z}, K(\mathbb{Z}, 2)=\times^{m} \mathbb{C} P^{\infty}$. This can be easily seen form the homotopy exact sequence of the Hopf fibration $S^{1} \rightarrow S^{2 n+1} \rightarrow \mathbb{C} P^{n}$ as $n \rightarrow \infty$. In this limit all the homotopy groups of $S^{2 n+1}$ vanish and so the only non-vanishing homotopy group of $\mathbb{C} P^{\infty}$ is $\pi_{2}\left(\mathbb{C} P^{\infty}\right)=\pi_{1}\left(S^{1}\right)=\mathbb{Z}$. However $\mathbb{C} P^{\infty}$ is also identified as $\mathrm{BU}(1)$, the universal classifying space of $S^{1}$ bundles. Thus $X_{3}$ is a fibration over $M$ which arises from gluing a fibre which is the "space of $S^{1}$ bundles" reminiscent of the gerbes according to [41]. It is also reminiscent of the emergence of $\mathbb{C} P^{2}$ in the exploration of the topological structure of $C_{M}^{\left[\omega^{3}\right]}$. These raise the question how $C_{M}^{\left[\omega^{3}\right]}$ is related to $X_{3}$, and whether the former can become a model for the latter. Clearly, this procedure for constructing Cspaces using Whitehead towers works for the rest of the cases involving higher degree forms.

For the special case of $M=S^{3}$ which is of interest in both DFT and theory of gerbes, the Whitehead fibration $\mathbb{C} P^{\infty} \rightarrow X_{3} \rightarrow S^{3}$ is a direct generalization of the Kaluza-Klein construction but now for the 3 -form which represents the generator of $H^{3}\left(S^{3}, \mathbb{Z}\right)$. In particular observe that $X_{3}$ and $S^{3}$ have the same homotopy groups for $n>3$ in direct analogy with the Kaluza-Kelin case where this statement is valid for $n>2$. It is tempting to assert that $X_{3}$ provides a homotopy representative for the gerbe.

It should also be noted that open strings with fixed origin geometrize all form fluxes. To see this, let $P_{*}(M)$ and $P(M)$ and be the space of paths in $M$ with and without fixed origin, respectively. There are two fibrations, $P_{*}(M) \rightarrow P(M) \rightarrow M$ and $\Omega_{*}(M) \rightarrow P_{*}(M) \rightarrow M$. The projection in the first fibration is defined as the point in $M$ that the path begins while the projection in the second fibration is the path end point projection. $P_{*}(M)$ is contractible as every path can be contracted to the origin. The first fibration implies that the configuration space of open strings is homotopic to the spacetime. The second fibration geometrizes all form fluxes. Indeed as $P_{*}(M)$ is contractible, the pull-back of all cohomology classes of the spacetime to $P_{*}(M)$ are cohomogically trivial.

\section{Concluding remarks}

We have proposed a C-space, $C_{M}^{\left[\omega^{3}\right]}$, for any closed 3-form $\omega^{k}$ on a manifold $M$ which represents a class $\frac{1}{2 \pi}\left[\omega^{3}\right] \in H^{3}(M, \mathbb{Z})$. These have been constructed by introducing appropriate new coordinates and after imposing suitable transition functions which are related to the transition functions of $M$ and the patching data of $\omega^{3}$ as arise in the Čech-de Rham theory. $C_{M}^{\left[\omega^{3}\right]}$ may not be manifolds. It is confirmed that $C_{M}^{\left[\omega^{3}\right]}$ satisfy the topological geometrization condition and provide a geometric explanation for a generalized geometry structure on the spacetime. The double spaces of DFTs are included as local subspaces in $C_{M}^{\left[\omega^{3}\right]}$. An interpretation of this is that for the global definition of DFTs additional coordinates are required. We argue that these new coordinates are necessary on topological grounds and this should not depend of the details of geometry. However how these can enter in the existing local description of DFTs remains an open problem.

We have also generalized the construction of C-spaces for any closed k-form on $M$, and we have established that $C_{M}^{\left[\omega^{k}\right]}$ have similar properties to those of $C_{M}^{\left[\omega^{3}\right]}$. It is expected that these spaces are required for the global definition of exceptional field theories. 
The construction of $C_{M}^{\left[\omega^{3}\right]}$ can be done starting from any spacetime with a good cover and a closed 3 -form. As a result such spaces can be found for all relevant supergravity backgrounds including those of the NS5-branes. Here we have explored in detail the 3 -torus with a 3-form flux model of [33]. We demonstrate how several puzzles associated with the construction of double spaces for this model [40] are resolved via the use of C-spaces.

Another method to topologically geometrize k-forms in the context of homotopy theory is that of Whitehead towers. It was emphasized that for simply connected manifolds and closed 2-forms, the Whitehead is related to the construction of the usual Kaluza-Klein space $C_{M}^{\left[\omega^{2}\right]}$ of circle fibrations. This raises the question whether $C_{M}^{\left[\omega^{3}\right]}$ can be also related to the Whitehead construction for 3-forms and in particular whether the former provide a model for the latter. Such a relation will elucidate the topological structure of C-spaces.

Although C-spaces resolve the global patching problem of double spaces, the additional coordinates enter linearly in the transition functions and so appear as too special to allow for a full covariance under all required symmetries, diffeomorphisms and dualities, without any further assumptions on the structure of spacetime. Nevertheless, they may prove to be useful way to proceed. In addition, the understanding how to incorporate the additional coordinates in DFT may lead to some new insights into the structure of these theories.

\section{Acknowledgments}

I would like to thank Jan Gutowski for many helpful discussions. I am partially supported by the STFC grant ST/J002798/1.

Open Access. This article is distributed under the terms of the Creative Commons Attribution License (CC-BY 4.0), which permits any use, distribution and reproduction in any medium, provided the original author(s) and source are credited.

\section{References}

[1] M.J. Duff, Duality rotations in string theory, Nucl. Phys. B 335 (1990) 610 [INSPIRE].

[2] A.A. Tseytlin, Duality symmetric closed string theory and interacting chiral scalars, Nucl. Phys. B 350 (1991) 395 [inSPIRE].

[3] W. Siegel, Two vierbein formalism for string inspired axionic gravity, Phys. Rev. D 47 (1993) 5453 [hep-th/9302036] [INSPIRE].

[4] W. Siegel, Superspace duality in low-energy superstrings, Phys. Rev. D 48 (1993) 2826 [hep-th/9305073] [INSPIRE].

[5] C.M. Hull, Doubled geometry and T-folds, JHEP 07 (2007) 080 [hep-th/0605149] [INSPIRE].

[6] O. Hohm, C. Hull and B. Zwiebach, Background independent action for double field theory, JHEP 07 (2010) 016 [arXiv: 1003.5027] [INSPIRE].

[7] O. Hohm, C. Hull and B. Zwiebach, Generalized metric formulation of double field theory, JHEP 08 (2010) 008 [arXiv: 1006.4823] [INSPIRE].

[8] I. Jeon, K. Lee and J.-H. Park, Differential geometry with a projection: application to double field theory, JHEP 04 (2011) 014 [arXiv:1011.1324] [INSPIRE]. 
[9] I. Jeon, K. Lee and J.-H. Park, Stringy differential geometry, beyond Riemann, Phys. Rev. D 84 (2011) 044022 [arXiv: 1105.6294] [INSPIRE].

[10] O. Hohm and S.K. Kwak, Frame-like geometry of double field theory, J. Phys. A 44 (2011) 085404 [arXiv:1011.4101] [InSPIRE].

[11] T. Kikuchi, T. Okada and Y. Sakatani, Rotating string in doubled geometry with generalized isometries, Phys. Rev. D 86 (2012) 046001 [arXiv:1205.5549] [INSPIRE].

[12] I. Vaisman, Towards a double field theory on para-Hermitian manifolds, J. Math. Phys. 54 (2013) 123507 [arXiv:1209.0152] [INSPIRE].

[13] I. Bakas and D. Lüst, 3-cocycles, non-associative star-products and the magnetic paradigm of R-flux string vacua, JHEP 01 (2014) 171 [arXiv:1309.3172] [INSPIRE].

[14] R. Blumenhagen, M. Fuchs, F. Haßler, D. Lüst and R. Sun, Non-associative deformations of geometry in double field theory, JHEP 04 (2014) 141 [arXiv: 1312.0719] [INSPIRE].

[15] B. de Wit and H. Nicolai, $d=11$ supergravity with local $\mathrm{SU}(8)$ invariance, Nucl. Phys. B 274 (1986) 363 [inSPIRE].

[16] P.C. West, $E_{11}$ and M-theory, Class. Quant. Grav. 18 (2001) 4443 [hep-th/0104081] [INSPIRE].

[17] F. Englert, L. Houart, A. Taormina and P.C. West, The symmetry of $M$ theories, JHEP 09 (2003) 020 [hep-th/0304206] [INSPIRE].

[18] C.M. Hull, Generalised geometry for M-theory, JHEP 07 (2007) 079 [hep-th/0701203] [INSPIRE].

[19] P.P. Pacheco and D. Waldram, M-theory, exceptional generalised geometry and superpotentials, JHEP 09 (2008) 123 [arXiv:0804.1362] [INSPIRE].

[20] C. Hillmann, Generalized $E_{7(7)}$ coset dynamics and $D=11$ supergravity, JHEP 03 (2009) 135 [arXiv:0901.1581] [INSPIRE].

[21] D.S. Berman and M.J. Perry, Generalized geometry and M-theory, JHEP 06 (2011) 074 [arXiv: 1008.1763] [INSPIRE].

[22] D.S. Berman, H. Godazgar, M. Godazgar and M.J. Perry, The local symmetries of M-theory and their formulation in generalised geometry, JHEP 01 (2012) 012 [arXiv:1110.3930] [INSPIRE].

[23] A. Coimbra, C. Strickland-Constable and D. Waldram, $E_{d(d)} \times \mathbb{R}^{+}$generalised geometry, connections and M-theory, JHEP 02 (2014) 054 [arXiv:1112.3989] [INSPIRE].

[24] A. Coimbra, C. Strickland-Constable and D. Waldram, Supergravity as generalised geometry II: $E_{d(d)} \times \mathbb{R}^{+}$and M-theory, JHEP 03 (2014) 019 [arXiv: 1212.1586] [INSPIRE].

[25] D.S. Berman, M. Cederwall, A. Kleinschmidt and D.C. Thompson, The gauge structure of generalised diffeomorphisms, JHEP 01 (2013) 064 [arXiv:1208.5884] [INSPIRE].

[26] M. Cederwall, J. Edlund and A. Karlsson, Exceptional geometry and tensor fields, JHEP 07 (2013) 028 [arXiv:1302.6736] [INSPIRE].

[27] H. Godazgar, M. Godazgar and H. Nicolai, Generalised geometry from the ground up, JHEP 02 (2014) 075 [arXiv:1307. 8295] [INSPIRE].

[28] O. Hohm and H. Samtleben, Exceptional field theory I: $E_{6(6)}$ covariant form of $M$-theory and type IIB, Phys. Rev. D 89 (2014) 066016 [arXiv:1312.0614] [INSPIRE]. 
[29] O. Hohm and H. Samtleben, Exceptional field theory. II. $E_{7(7)}$, Phys. Rev. D 89 (2014) 066017 [arXiv: 1312.4542] [INSPIRE].

[30] G. Aldazabal, M. Graña, D. Marqués and J.A. Rosabal, The gauge structure of exceptional field theories and the tensor hierarchy, JHEP 04 (2014) 049 [arXiv: 1312.4549] [INSPIRE].

[31] G. Aldazabal, D. Marques and C. Núñez, Double field theory: a pedagogical review, Class. Quant. Grav. 30 (2013) 163001 [arXiv:1305.1907] [INSPIRE].

[32] D.S. Berman and D.C. Thompson, Duality symmetric string and M-theory, Phys. Rept. 566 (2014) 1 [arXiv: 1306.2643] [InSPIRE].

[33] O. Hohm, D. Lüst and B. Zwiebach, The spacetime of double field theory: review, remarks and outlook, Fortsch. Phys. 61 (2013) 926 [arXiv:1309.2977] [INSPIRE].

[34] O. Hohm and B. Zwiebach, Large gauge transformations in double field theory, JHEP 02 (2013) 075 [arXiv:1207.4198] [INSPIRE].

[35] D.S. Berman, M. Cederwall and M.J. Perry, Global aspects of double geometry, JHEP 09 (2014) 066 [arXiv:1401.1311] [INSPIRE].

[36] C.M. Hull, Finite gauge transformations and geometry in double field theory, JHEP 04 (2015) 109 [arXiv:1406.7794] [INSPIRE].

[37] M. Cederwall, The geometry behind double geometry, JHEP 09 (2014) 070 [arXiv: 1402.2513] [INSPIRE].

[38] H. Godazgar, M. Godazgar and H. Nicolai, Einstein-Cartan calculus for exceptional geometry, JHEP 06 (2014) 021 [arXiv:1401.5984] [INSPIRE].

[39] E. Cartan, Les groupes des transformations continues, infinis, simples (in French), Ann. Sci. Ecole Norm. Sup. 26 (1909) 93.

[40] G. Papadopoulos, Seeking the balance: patching double and exceptional field theories, JHEP 10 (2014) 089 [arXiv:1402.2586] [InSPIRE].

[41] N.J. Hitchin, Lectures on special Lagrangian submanifolds, math/9907034 [INSPIRE].

[42] J.-L. Brylinski, Loop spaces, characteristic classes and geometric quantization, chapter 5, Progr. Math. 107, Birkhäuser, Boston U.S.A. (1993).

[43] N. Hitchin, Generalized Calabi-Yau manifolds, Quart. J. Math. 54 (2003) 281 [math/0209099] [INSPIRE].

[44] N. Hitchin, Brackets, forms and invariant functionals, math/0508618 [INSPIRE].

[45] M. Gualtieri, Generalized complex geometry, Ph.D. thesis, Oxford University, Oxford U.K. (2003) [math.DG/0401221].

[46] R. Bott and L.W. Tu, Differential forms in algebraic topology, Springer-Verlag, Heidelberg Berlin Germany and New York U.S.A. (1982).

[47] D. Husemoller, Fibre bundles, Graduate Texts in Mathematics, Springer-Verlag, Heidelberg Berlin Germany and New York U.S.A. (1966). 